Meta

Journal des traducteurs

Translators' Journal

\title{
Symbolique animale et traduction
}

\section{Jean Tournier}

Volume 31, numéro 3, septembre 1986

Prismes de traductions littéraires

Facets of Literary Translation

URI : https://id.erudit.org/iderudit/004501ar

DOI : https://doi.org/10.7202/004501ar

Aller au sommaire du numéro

Éditeur(s)

Les Presses de l'Université de Montréal

ISSN

0026-0452 (imprimé)

1492-1421 (numérique)

Découvrir la revue

Citer cet article

Tournier, J. (1986). Symbolique animale et traduction. Meta, 31(3), 332-349.

https://doi.org/10.7202/004501ar

Ce document est protégé par la loi sur le droit d'auteur. L'utilisation des services d'Érudit (y compris la reproduction) est assujettie à sa politique d'utilisation que vous pouvez consulter en ligne.

https://apropos.erudit.org/fr/usagers/politique-dutilisation/
Cet article est diffusé et préservé par Érudit.

Érudit est un consortium interuniversitaire sans but lucratif composé de l’Université de Montréal, l'Université Laval et l'Université du Québec à Montréal. Il a pour mission la promotion et la valorisation de la recherche. https://www.erudit.org/fr/ 


\section{SYMBOLIQUE ANIMALE ET TRADUCTION*}

JEAN TOURNIER

UNIVERSITÉ DE FRANCHE-COMTÉ, BESANÇON, FRANCE

La symbolique animale est l'un des éléments culturels de toute communauté linguistique. Dans chaque culture, les animaux les plus courants (moins de 200) sont considérés comme symbolisant une ou plusieurs particularités (défauts, qualités, caractéristiques physiques ou comportementales). Ces particularités peuvent être réelles, mais elles peuvent également être prêtées par la tradition aux animaux, à qui l'on attribue des particularités humaines que l'on croit - ou que l'on a cru - reconnaître en eux. Le paradoxe de la symbolique animale est qu'elle implique fondamentalement une vision anthropocentrique.

Pour le traducteur, comme pour l'anthropologue, l'intérêt de la symbolique animale est qu'elle diffère partiellement d'une communauté à l'autre. Ainsi, le lézard symbolise la paresse aussi bien dans la symbolique française que dans la symbolique anglaise, mais symbolise l'absence de modestie dans la symbolique ibo (Nigéria). La vache, animal sacré en Inde, symbolise pour les anglophones la vulgarité et, pour les francophones, le caractère "bête et méchant " attribué traditionnellement aux gendarmes ("Mort aux vaches!"). La bonne santé, symbolisée en français par le gardon, est symbolisée par le porc en arabe maghrébin. Le pélican, qui en France est symbole d'abnégation, symbolise la fourberie dans le nord-ouest de l'Inde (Gujerat). Le serpent qui, dans les civilisations judéo-chrétiennes, notamment, symbolise les forces du mal, symbolisait, pour les Latins et les Grecs - du fait de la mue qui le caractérise - le renouveau de la vie, d'où sa présence sur le caducée, lui-même symbole de la médecine, qui est censée guérir le malade et, par conséquent, lui redonner une nouvelle vie : le serpent était l'attribut d'Esculape, dieu de la médecine et de la guérison, et non pas l'incarnation du dieu des enfers.

La faune spécifique d'un pays a, évidemment, un rôle dans la constitution de la symbolique animale d'une communauté : si, en arabe maghrébin, on dit littéralement "sauter comme une gerboise ", on ne s'étonnera pas que la comparaison soit différente dans les pays où la gerboise est peu connue : cette expression arabe peut se rendre en français par "sauter comme un cabri " ou "faire des cabrioles " (cabri étant le synonyme méridional de chevreau). Ceci explique également que dans les variétés d'une même langue, correspondant à des environnements distincts, il puisse y avoir des différences dans la symbolique animale : le nom du bison (buffalo) n'apparait pas plus dans la symbolique de l'anglais britannique que l'animal en question n'apparaît dans la Plaine de Salisbury, alors qu'il a logiquement sa place dans la symbolique de l'anglais d'Amérique (to buffalo = to keep in awe). Mais tout ne dépend pas de la faune locale et il y a osmose entre les cultures : le tigre symbolise la jalousie, même dans les campagnes françaises.

D'autre part, il faut tenir compte, dans les variétés d'une même langue, des usages lexicaux spécifiques. Ainsi, le mot jackass est interprété par les Britanniques comme désignant l'âne mâle, symbole de la sottise. Mais en Australie, ce mot est interprété dans le sens de laughing jackass, qui est une sorte de grand martin-pêcheur (oiseau que les abo- 
rigènes appellent kookaburra). Le mot jackass n'apparaît donc pas, dans la symbolique de l'anglais d'Australie, dans le sens d'" âne ", même si l'âne, lui, y apparaît, plus spécialement sous la forme donkey. (Le tabou qui porte sur ass du fait de sa confusion avec la forme réduite de arse tend à exclure le mot ass de la symbolique, mais non pas l'animal correspondant.)

Toutefois, le traducteur est évidemment moins concerné par les différentes variétés géographiques d'une même langue que par la transposition d'une langue à une autre. S'agissant de la symbolique animale, il devra veiller aux adaptations indispensables. Par exemple, ci cuckoo symbolise la folie en anglais, ce n'est pas le cas pour coucou en français, et "traduire" le titre du film de Milos Forman "One fight over a cuckoo's nest" par "Vol au-dessus d'un nid de coucou ", comme cela a été fait, est une aberration : aucun francophone, du moins en Europe, ne comprend que " nid de coucou " suggère un hôpital psychiatrique. Pour citer un autre exemple, nos étudiants de deuxième année ont eu à traduire un passage des Célibataires, de Montherlant, où il était question d'" une charmante musaraigne féminine ", façon de suggérer simplement le profil un peu " pointu " de la jeune femme. Les meilleurs étudiants ont perçu que le mot musaraigne suggérait quelque chose de tout à fait différent de ce que suggère l'équivalent shrew en anglais (cf. la pièce de Shakespeare The Taming of the Shrew). Traduire musaraigne par shrew était donc une erreur (d'autant plus qu'une mégère ne peut guère être « charmante "). Il fallait utiliser le nom d'un autre animal à profil pointu, en évitant le putois, car polecat suggère la mauvaise odeur. Certains ont intelligemment proposé de changer la musaraigne en belette (weasel; $\mathrm{cf}$. weasel-faced). Le traducteur, tel l'enchanteur Merlin, a le pouvoir (et le devoir) d'opérer des métamorphoses.

Dans le chapitre VI - qui traite de la métasémie - de notre Introduction descriptive à la lexicogénétique de l'anglais contemporain (Paris-Genève, Champion-Slatkine, 1985), nous avons consacré quelques pages à la symbolique animale (pp. 238-243). Mais il s'agissait alors d'une illustration de la métaphore zoomorphique, du point de vue du sémanticien. Nous envisageons ici la question d'un point de vue différent : celui du traducteur, pour qui le passage de la symbolique d'une communauté linguistique à la symbolique d'une autre communauté linguistique entraîne forcément quelques difficultés.

En fait, si l'on approfondit les quelques observations faites jusqu'ici dans cet article, on s'aperçoit à l'analyse que ces difficultés - d'ailleurs mineures et assez aisément surmontables - proviennent d'une triple inadéquation des symboliques entre elles.

a) Tout d'abord, du point de vue de la nomenclature, le découpage du règne animal n'est pas le même dans les deux langues, et est souvent plus « fin » en anglais. Ainsi, le français singe ne fait pas la distinction que fait l'anglais entre ape et monkey, ni tortue la distinction faite entre tortoise et turtle, ni papillon la distinction faite entre butterfly et moth, ni serpent la distinction faite entre snake et serpent, ni vipère la distinction faite entre viper et adder. L'inadéquation du découpage est évidemment plus gênante si chacun des deux noms d'animaux anglais correspond à un symbole différent : butterfly symbolise l'insouciance et la frivolité et moth l'attirance des objets clinquants et des fausses valeurs ; adder symbolise la surdité et viper la traîtrise et la méchanceté.

b) Ensuite, la liste même des animaux constituant la symbolique n'est pas la même dans les deux communautés. Ainsi, la couleuvre, le merle, le phoque, le cloporte, le cafard, la dinde, qui appartiennent à la symbolique française, n'appartiennent pas à la symbolique anglaise. Inversement, la musaraigne (shrew), le jars (gander), l'épaulard (grampus), la foulque (coot), le dronte (dodo), le pluvier (plover), qui appartiennent à la symbolique anglaise, n'appartiennent pas à la symbolique française.

c) Enfin, si l'on considère uniquement les animaux communs aux deux symboliques, on remarque que les symboles attachés à chaque animal, s'ils peuvent être rigou- 
reusement identiques, peuvent être aussi, soit partiellement, soit totalement, différents. Ainsi, le crocodile symbolise l'hypocrisie en français (" larmes de crocodile ") aussi bien qu'en anglais ("crocodile tears") ou en espagnol ("lágrimas de cocodrilo"). Mais le porc (ou cochon), s'il symbolise la saleté physique en français comme en anglais, symbolise en plus en anglais l'égoïsme (cf. "male chauvinist pig ") et symbolise en plus en français la lascivité (cf. "un vieux cochon"). La chatte symbolise en anglais la méchanceté et l'agressivité féminines, un peu comme la musaraigne (cf. "she's a cat ») et, en français, le caractère caressant (cf. " elle est très chatte").

Une étude systématique, portant sur les quelque 160 noms d'animaux qui forment l'essentiel de la symbolique animale de l'anglais, permet de se faire une idée précise des analogies et des différences des deux symboliques et de la nécessité des transpositions qu'entraînent ces différences pour le traducteur. Il suffit, pour réaliser cette étude, de réunir puis d'analyser l'ensemble des métaphores et des comparaisons lexicalisées où apparaissent des noms d'animaux.

Précisons qu'en anglais ce type de métaphore se manifeste surtout de quatre façons :

- emploi du nom de l'animal dans un sens dérivé : " he's a bear ; she's a goose ";

- emploi du nom de l'animal dans un composé : pig-headed;

- emploi du nom de l'animal converti en verbe : "he's beavering away»;

- emploi d'un mot suffixé dérivé du nom de l'animal, ce mot suffixé étant un adjectif (mulish) ou un nom (jackassery).

Quant aux comparaisons, elles se manifestent surtout de deux façons :

- avec like, soit selon la formule générale $\mathrm{V}+$ like + Nom d'animal (eat like a horse), soit, beaucoup plus rarement, avec like en position à droite dans un adjectif composé ( $a$ bear-like gait »), voire, exceptionnellement, sans like, dans une lexie composée, prépositionnelle ou complexe (at a snail's pace).

- avec $a s$, selon la formule générale (as) + Adjectif + as + Nom d'animal ((as) merry as a grig). Très accessoirement, la comparaison peut s'exprimer à l'aide du suffixe -wise (crabwise).

Dans l'analyse qui suit, nous classerons les animaux en quatre catégories : mammifères ( 69 noms) ; oiseaux ( 41 noms) ; poissons, mollusques, reptiles, batraciens, crustacés, annélides ( 29 noms); insectes, arachnides (22 noms).

Dans chaque catégorie, les noms anglais seront classés alphabétiquement. Nous utiliserons les sigles SA et SF respectivement pour symbolique anglaise et symbolique française.

\section{MAMMIFÈRES}

$A P E-$ Le singe anthropoïde symbolise l'imitation en SA et SF (to ape $=$ singer, cf. all. nachäffen) ; également en SA l'homme fruste, brutal : big ape peut se rendre en fr. par primate. Pour les autres symboles du fr. singe (méchant, malin, bavard), voir MONKEY.

ASS (DONKEY, JACKASS) - L'âne est symbole de sottise et d'ignorance en SA et SF, avec une nuance de ridicule en SA. (Dire des âneries = talk rubbish.) L'entêtement, que l'âne symbolise aussi en SF est plutôt attribué à $D O N K E Y$ qu'à $A S S$, d'où l'expression (vieillie) to ride the black donkey = être têtu comme une mule. DONKEY est également en SA un symbole de longévité, figé dans not for donkey's years, une tradition ancienne voulant qu'on ne voie jamais d'âne mort. JACKASS, $\mathbf{N}=$ bête, Adj. ; jackassery = bêtise(s). 
$B A B O O N$ - Le babouin, absent en SF, symbolise la laideur en SA (fr. laid comme un singe), ainsi que le caractère fruste, sans culture (fr. plouc, péquenot).

$B A T$ - La chauve-souris symbolise la quasi-cécité ou la myopie en SA : blind as a bat; correspond à la taupe en SF (myope comme une taupe). Egalement en SA, dans les adj. batty et bats, BAT symbolise la folie (have bats in the belfry; cf. fr. avoir une araignée au plafond).

$\boldsymbol{B E A R}$ - L'ours symbolise en SA et en SF le caractère peu sóciable, bourru, la mauvaise humeur ; aux États-Unis, il symbolise aussi la forte carrure : a bear of a man= une armoire à glace, un grand balèze.

$B E A S T$ - En SA symbolise la brutalité et la méchanceté, mais non pas la bêtise ( = fr. brute; vache, chameau). Mais die like a beast se traduit litterralement.

$\boldsymbol{B E A} \boldsymbol{V E R}$ - Le castor, absent en SF, symbolise l'assiduité au travail, d'où eager beaver (= bourreau de travail) et le verbe beaver $(=$ bosser $)$.

$B I T C H$ - En SA et SF, la chienne symbolise la prostitution et la nymphomanie, mais le symbole est plus courant en SA : son of a bitch se rend par fils de pute. BITCH symbolise également la méchanceté, assez couramment en SA, mais très rarement en SF, et, dans ce sens, se rend plutôt par rosse, vache, chameau. S'applique aussi aux non-animés : "that bitch of $a$ job" = "ce foutu boulot ». Le verbe bitch se rend par rouspéter, râler.

BOAR - Le sanglier (wild boar) est pratiquement absent de SF et relativement rare en SA où il symbolise la brutalité. (The Boar était le surnom de Richard III.)

BUFFALO - Absent en SF, le bison apparaît en anglais d'Amérique sous la forme du verbe, dans le sens d'« intimider, impressionner volontairement".

$B U L L-E n$ SA et SF, le taureau symbolise la force brutale exercée sans discernement : go at it like a bull at a gate = foncer tête baissée. Accessoirement, en SA et SF, $B U L L$ peut symboliser la vigueur sexuelle. De plus, en SA, il symbolise la maladresse, la balourdise, dans like a bull in a china shop (se rend ici par éléphant).

$B U L L D O G$ - Le bouledogue, parfois utilisé en SF comme symbolisant l'abord revêche, est en SA symbole de ténacité, d'acharnement : "he is one of the bulldog breed "se rend sans métaphore : "il est d'une ténacité à toute épreuve ".

CALF - En SA et SF symbole de balourdise, de maladresse, d'un tempérament " empoté " et manquant de ressort, le veau est en plus, en SA, symbole de jeunesse et d'inexpérience : calf-love = amours juvéniles. Il symbolise aussi en SA un caractère inoffensif : as meek as a calf (ou as a lamb) = doux comme un agneau. Pleurer comme un veau se rend par cry one's heart out, qui perd la connotation ironique.

CAMEL - En SF, mais non pas en SA, le chameau est symbole de méchanceté : on peut rendre le nom chameau par l'adjectif beastly. Moins couramment, mais en SA comme en SF, il symbolise la sobriété et l'endurance, et, plus rarement, en SA, la soumission.

CAT - Le chat (ou la chatte) est symbole en SA de la méchanceté et de l'agressivité féminines. Chatte est utilisé en fr. comme adjectif dans le sens de " caressant " (angl. kittenish). Un jeu de mots comme "His kittenish bride has grown into a cat" peut se rendre approximativement par "Sa jeune épouse, qui était très chatte, s'est changée en tigresse " (ou " en chat sauvage "). A noter aussi (as) sick as a cat = malade comme un chien.

$C A T$ and $D O G$ - La séquence chien et chat (dans cet ordre en fr., en esp., et en all.) symbolise la mésentente, la rivalité, la querelle, en SF comme en SA. 
COW - La vache symbolise la méchanceté sotte en SF. C'est aussi le cas, mais bien moins fréquemment (et seulement pour la femme) en SA, où cet animal symbolise plutôt la vulgarité. Ainsi, "c'est une vache " ne peut se traduire littéralement que s'il s'agit d'une femme, alors que l'expression française désigne couramment un homme. En espagnol, l'expression "parece une vaca" ne concerne que l'aspect physique (= "c'est une grosse dondon"). Un cheval peut donner "a cow kick" (= "un coup de pied en vache»).

COYOTE - Peu courant en SF, le coyote symbolise la fripouille, le vaurien. Cette métaphore s'est répandue à partir des États-Unis, grâce aux westerns.

DALMATIAN - Absent en SF, le dalmatien symbolise en SA l'aspect tacheté (cf. son nom populaire de spotted Dick). Les équivalents possibles en SF, maquereau, léopard, grive (cf. grivele), sont très rarement utilisés dans ce sens.

DEER - Ce terme générique désignant l'espèce des cervidés symbolise en SA l'agilité, la célérité : as swift as a deer peut être rendu en fr. par rapide comme une gazelle ou vif comme l'éclair.

$D O E$ - Porteur du même symbole que DEER en SA, ce terme désigne la femelle des cervidés (sauf la femelle adulte du cerf, désignée par hind) et celle des léporidés. Le composé doe eyes (= yeux de biche) symbolise le regard innocent et soumis. (N.B. Le verbe fawn (= faire le chien couchant) n'a aucun rapport avec le nom fawn, désignant le faon, jeune cervidé.)

$D O G$ - Le chien est porteur de plusieurs symboles, en SF comme en SA, mais ils ne se recoupent pas exactement. Les symboles communs sont la fidélité soumise (suivre qn comme un petit chien $=$ to dog s.o. 's footsteps) et la mauvaise qualité : chien de temps, temps de chien (cf. esp. tiempo de perros ; all. Hundewetter) ; vie de chien = a dog's life (cf. esp. "que vida mas perra!"); et aussi dog-latin (= latin de cuisine), dog-rose (même métaphore que cynorhodon), not a dog's chance. Moins fréquemment, en SF, le chien symbolise l'intrusion maladroite dans " un chien dans un jeu de quilles" (cf. angl. a bull in a china-shop) et la ladrerie : "il est chien " = «il est radin ». En SA, il peut représenter l'homme en général : lucky dog, dirty dog, every dog has his day, et aussi symboliser la gourmandise et la voracité : as greedy as a dog. Dans dog eat dog, il correspond au loup en SF.

DORMOUSE - En SA comme en SF, le loir symbolise le sommeil profond.

ELEPHANT - En SF, l'éléphant est symbole de maladresse, de caractère "empoté ", dû notamment à l'énormité de la taille : cf. " un éléphant dans un magasin de porcelaine" (rendu par $B U L L$ ). En SF et en SA, il symbolise la mémoire. L'éléphant blanc, en SF, symbolise plutôt la rareté, et, en SA, le caractère superflu et encombrant d'un objet. Les éléphants roses n'existent pas (encore) en SF, mais l'expression anglaise "see pink elephants" suggère l'ivresse, voire le delirium tremens.

FOX - Le renard symbolise la ruse, en SA comme en SF et ailleurs (cf. esp. astuto como un zorro - voir aussi le personnage de Zorro, mais hacerse el zorro = faire l'imbécile). En plus, en SA, il symbolise la couleur rousse : le surnom Foxy peut se rendre par Poil de carotte (cf. all. fuchsig, au sens propre).

GOAT - En SF et SA, le mâle (bouc = billy-goat ou he-goat) symbolise la puanteur. En plus, en SA, il symbolise la lubricité : " an old goat" = "un vieux cochon"; the goat gad = le dieu Pan. La femelle (chèvre = nanny-goat ou she-goat) est en SF symbole de sottise, d'abrutissement (f. "il me fera devenir chèvre") et, en SA, 
porte un symbole voisin, le caractère écervelé : act the (giddy) goat = faire l'andouille.

GRAMPUS - L'épaulard, absent en SF, symbolise en SA la respiration bruyante, l'essoufflement : puff and blow like a grampus = souffler comme un phoque (ou comme un bouff).

$H A R E$ - Le lièvre symbolise la couardise en SA, en SF et ailleurs (cf. all. furchtsam wie ein Hase) et accessoirement la vitesse : run like a hare (mais on dit plutôt en fr. détaler comme un lapin). Le composé hare-brained (= écervelé, tête de linotte) rappelle un peu l'expression française vieillie une mémoire de lièvre (c'est-à-dire une mauvaise mémoire). March hare symbolise la folie.

HEDGEHOG - Le hérisson, très peu utilisé en SF, symbolise en SA l'insociabilité, comme le fait l'ours.

HORSE - Le cheval, en SA et SF, symbolise la force au travail (cf. aussi all. wie ein Pferd arbeiten). En SF, il symbolise également la femme grande, plutôt forte et assez peu féminine : cf. " c'est un vrai cheval " (même métaphore en espagnol), c'està-dire un certain manque de " race ", de finesse physique, alors qu'en SA il symbolise le manque de raffinement comportemental, une certaine grossièreté : cf. horselaugh (= gros rire), horseplay (= "jeux brutaux "), to horse about (= chahuter, "jouer brutalement "). Enfin, en SA, le cheval symbolise le gros mangeur : eat like $a$ horse = manger comme un ogre, ou comme quatre.

HOUND - Pratiquement absent en SF, le chien de chasse a une valeur péjorative en $\mathrm{SA}:$ : he's an unmitigated hound" = "c'est un sale type ". Bloodhound, comme son équivalent français limier, ou souvent "fin limier", désigne le détective, l'enquêteur de la police.

HYENA - L'hyène symbolise en SF l'agressivité (surtout féminine) et, en SA, la cruauté doublée de traîtrise. To laugh like a hyena évoque le rire satanique.

$\boldsymbol{J A C K A L}$ - Symbole du profiteur cruel et sans scrupule en SF, le chacal symbolise en SA la complicité servile.

$K I D$ - Le chevreau, absent en SF, symbolise l'enfance en SA. Kid s'utilise aussi, traditionnellement, en anglais, comme surnom de boxeur.

KITTEN - Le chaton, pratiquement absent en SF, symbolise en SA le caractère "joueur ", taquin, d'une part (as playful as a kitten) et caressant, affectueux, d'autre part (kittenish). On trouve aussi as weak as a kitten.

$\boldsymbol{L A M B}$ - L'agneau symbolise la douceur innocente, la docilité, en SA comme en SF et ailleurs (cf. esp. manso como un cordero; all. fromm wie ein Lamm).

LEOPARD - Absent ou très rare en SF, le léopard symbolise en SA l'aspect tacheté, comme le dalmatien (cf. le proverbe : the leopard cannot change his spots = chassez le naturel : il revient au galop).

LION - Le lion est symbole de courage, en SA comme en SF et ailleurs (cf. esp. valiente como un león; all. löwenmutig). Il symbolise également le roi, le chef noble, d'où la part du lion (= the lion's share). Mais en SA il symbolise en plus, d'une part, la célébrité (d'où lion-hunter, lionize, qui se rend en fr. par une périphrase) et, d'autre part, la Grande-Bretagne (to twist the lion's tail = "narguer les Britanniques"). Dans ce dernier cas, le français doit préciser "le lion britannique ". A noter aussi : to put one's head in the lion's mouth = se jeter dans la gueule du loup. 
$L Y N X$ - En SA comme en SF, le lynx symbolise la vue perçante.

MAMMOTH - Dans la culture française, le mammouth évoque immanquablement une chaîne de magasins à grande surface. En SA, il symbolise l'énormité de la taille, le gigantisme, et s'utilise comme adjectif (cf. fr. monstre).

MARE - Absente en SF, la jument apparait surtout en SA dans the grey mare is the better horse (= "c'est la femme qui porte la culotte"). A mare's nest symbolise quelque chose d'illusoire : find a mare's nest est faire ce que l'on croit être une découverte mais qui, en réalité, n'en est pas une.

MOLE - En SF comme en SA, la taupe symbolise la quasi-cécité, la myopie, comme la chauve-souris en SA. Le composé français une vieille taupe peut se rendre par an old cat.

MONKEY - La symbolique du singe en SF se répartit en SA entre APE et MONKEY. $M O N K E Y$ symbolise en SA, comme singe en SF, la malignité, le manque de franchise (malin comme un singe = as mischievous as a monkey ; cf. aussi monkey business, monkey tricks et même to monkey about with sth = "tripoter quelque chose qu'on ne doit pas toucher "). Il symbolise également, en SF et en SA, la loquacité, le bavardage, d'une part, et, d'autre part, l'agilité (cf. aussi all. flink wie ein $A f f e$ ).

MOUSE - La souris symbolise, en SF et en SA, la discrétion, la timidité, le calme, voire, en SA le mutisme (as mute as a mouse). L'adj. mousy peut se rendre par effacé.

$M U L E$ - En SA et en SF, la mule (ou le mulet) symbolise l'obstination, l'entêtement.

$\boldsymbol{O X}$ - En SA et en SF, le bœuf symbolise la force tranquille, avec, en SF, un sens supplémentaire, celui de "colossal " (un succès bœuf; un effet bœuf), d'emploi un peu vieilli. En SA, il symbolise aussi, d'une part, la patience (as patient as an ox) et, d'autre part, la balourdise (" he's a big ox" = "c'est un gros balourd ").

PANTHER - Pratiquement absente en SF, la panthère symbolise en SA la souplesse et l'agilité (as lithe as a panther = souple comme une liane).

PIG (HOG, SWINE) - Le porc (ou cochon) symbolise, en SF comme en SA, la saleté physique, d'une part (fr. cochonner, porcherie; angl. to pig together, to hog it) et, d'autre part, l'entêtement (fr. tête de cochon; angl. pig-headed, as wilful as a pig). La "saleté morale " prend, en SF, une connotation érotique (cf. cochon qui sommeille, histoire cochonne, exceptionnelle en SA, où le porc symbolise plutôt la bassesse, la veulerie méchante ( $a$ swine = un salaud, une sombre vache). Par ailleurs, le porc symbolise en SA, mais non pas en SF, l'égoïsme, d'une part, et la goinfrerie, d'autre part (make a pig of oneself = bâfrer; " he's a greedy hog "). En SF, cet animal peut symboliser, comme le chien, la mauvaise qualité (cf. travail de cochon, temps de cochon, cochon de temps, symbolique transférée à la truie en allemand : Sauarbeit, Sauwetter). Hog symbolise plutôt la goinfrerie, l'égoïsme et l'absence d'éducation (cf. fr. pourceau et angl. roadhog = chauffard).

POLECAT - En SA, le putois symbolise la puanteur : stink like a polecat se rend par puer comme un bouc. En SF, on ne trouve guère que l'expression gueuler comme un putois = to screech like a peacock.

$P U P, P U P P Y$ - Le chiot symbolise en SA et en SF le caractère remuant, maladroit et pataud. En SA, il symbolise aussi la jeunesse inexpérimentée (puppy love = les premières amours), mais aussi prétentieuse ( $a$ young pup $=$ un blanc-bec, un petit morveux). 
$\boldsymbol{R A B B I T}$ - Le lapin, comme le lièvre, symbolise, en SA et en SF, la couardise et la rapidité dans la course, et aussi la fécondité, la prolifération. Il n'y a pas d'équivalent littéral à chaud lapin (= randy devil), ni à poser un lapin à qn (to stand $s b u p$ ).

$R A C O O N$ - Absent en SF, le raton-laveur symbolise en SA le Noir, surtout sous la forme aphétique coon. Le mot, d'origine américaine, est vulgaire et raciste.

$\boldsymbol{R} \boldsymbol{A T}$ - Symbole en SF de mesquinerie, d'avarice, mais peu usité (et peut-être influencé par radin et rapiat), le rat symbolise en SA l'individu lâche, fourbe, méprisable, d'où shake like a rat, to rat (= retourner sa veste), to rat on a pal (= vendre un copain), et le sens "syndical " de rat, non-gréviste. Le composé rat-race symbolise l'âpre concurrence pour se faire " une place au soleil ". I smell a rat peut se rendre par il y a anguille sous roche, et drunk as a rat par soûl comme une bourrique.

SHEEP - En SA et en SF, le mouton symbolise l'instinct grégaire. En SF, il symbolise aussi la docilité, la douceur, comme $L A M B$ en SA. Make sheep's eyes at correspond à faire les yeux doux à (des yeux de merlan frit, en langue populaire). En argot des prisons, un mouton est un mouchard, un indicateur ( $=$ stool pigeon). The black sheep (cf. all. das schwarze Schaf) se rend par la brebis galeuse.

SHREW - Absente en SF, la musaraigne symbolise en SA l'agressivité, la hargne féminines.

SKUNK - Absente en SF, la mouffette symbolise en SA, d'une part, la puanteur, et, d'autre parţ, le caractère méprisable en général et la muflerie masculine en particulier.

SLOTH - Absent en SF, le paresseux, édenté tardigrade d'Amérique, est en SA symbole d'indolence (ce qui est précisément l'autre sens de ce mot, dérivé de slow).

SoW - Pratiquement absente en SF, sauf pour désigner vulgairement une grosse femme prolifique, la truie n'apparaît en anglais que dans quelques rares expressions comme as drunk as David's sow, ou a pig of his own sow (= la monnaie de sa pièce).

SPANIEL - Absent en SF, l'épagneul symbolise en SA l'obséquiosité.

SQUIRREL - En SF, symbole de l'épargne et de la prévoyance, d'une part, et de l'agilité, d'autre part, l'écureuil est porteur des mêmes symboles en SA, mais le second est beaucoup plus utilisé que le premier : as nimble as a squirrel (cf. aussi esp. listo como una ardilla).

$S T A G$ - En SF, le cerf symbolise surtout la rapidité, comme $D E E R$ en SA. En SA, il symbolise l'homme, par opposition à la femme (cf. stag-party \# hen-party) et, aux États-Unis, 1'homme qui va à une réception ou à une soirée sans compagne. L'ancien symbole de cerf en SF et de stag en SA (cornard, cocu) tend à disparaître.

TIGER - Le tigre symbolise la jalousie couramment en SF, rarement en SA. Il est surtout, en SA et en SF, symbole de férocité (tigerish = sanguinaire), souvent désignant une femme : "she fought like a tiger" = " elle s'est battue comme une tigresse ». La référence à l'aspect rayé du pelage apparaît en anglais dans tiger-lily, et en français dans chat tigré = tabby cat (où tabby vient du nom d'un quartier de Bagdad où on fabriquait un taffetas à rayures).

WALRUS - Absent en SF, le morse n'apparaît en SA que dans le composé humoristique walrus-moustache (= moustache à la gauloise).

WHALE - Absent en SF (sauf exceptionnellement pour désigner ironiquement une femme grande et corpulente), la baleine symbolise l'énormité de la taille et ce mot 
a, par extension, une valeur intensive : $a$ whale of a lot ; " there's $a$ whale of a difference " = "c'est vachement différent ".

WOLF - En SF et plus encore en SA, le loup symbolise la voracité (cf. fr. une faim de loup; angl. eat like a wolf, to wolf down, wolfish). En SA et plus encore en SF, il symbolise aussi la férocité. De plus, en SA, il symbolise le tombeur de femmes, symbole que l'on ne trouve en français que de façon épisodique, dans des expressions vieillies comme "prends garde au loup ", " elle a vu le loup ". Mais la psychanalyse des contes de fées rend clair ce symbole du loup. À noter aussi : mettre le loup dans la bergerie = to set the fox to mind the geese, et les loups ne se mangent pas entre eux $=$ dog does not eat dog.

ZEBRA - Le zèbre symbolise l'aspect rayé, en SA et en SF (cf. fr. zébrer, zébrure ; angl. zebra-crossing). Un drôle de zèbre = a queer bird.

\section{OISEAUX}

BANTAM COCK - Absent en SF, le coq nain symbolise le "cran » en SA : as plucky as a bantam cock.

$B I R D$ - Comme le mot oiseau dans un drôle d'oiseau, le mot $B I R D$ peut désigner un individu quelconque, souvent précisé par le déterminant : a queer bird, a gallowsbird, a cunning old bird. Il symbolise aussi la jeune femme en SA (fr. une nana). D'autre part, il symbolise en SA et en SF le manque d'appétit (eat like a bird) et le caractère écervelé (avoir une cervelle d'oiseau = to be bird-witted), ainsi que la gaîté, mais plus rarement (to sing like a bird). Birds ans bees (comme the facts of life) constitue un euphémisme symbolisant les phénomènes de la sexualité et de la reproduction, tels qu'ils doivent être révélés aux enfants.

BUZZARD - Symbole de sottise en SF, la buse est symbole de myopie en SA (as blind as a buzzard).

CHICKEN - Le mot poulet, paronyme approximatif de police, symbolise le policier en français populaire. En SA, le poulet symbolise, d'une part, la jeunesse tendre : as tender as chicken (cf. aussi son emploi comme terme affectueux : chicken, chick = mon poussin, mon poulet, ma poule, mon coco, ma cocotte), et, d'autre part, le caractère peureux : chicken-hearted, chicken-liver (cf. fr. poule mouillée); to chicken out $=$ se dégonfler.

COCK - En SA et en SF, le coq symbolise la hardiesse (relative et un peu ridicule) : as bold as a cock on his own dunghill. (Dans cocksure, cock est un substitut paraphonique de God. Quant à l'emploi vulgaire de cock dans le sens de "pénis ", il représente une métaphore dérivée d'une première métaphore : $\operatorname{cock}=$ "robinet ").

COOT - Absente en SF, la foulque symbolise en SA la calvitie : bald as a coot et, accessoirement la sottise.

CROW - Absente en SF, la corneille symbolise, d'une part, la noirceur physique (as black as a crow), comme le corbeau en français, et, d'autre part, l'enrouement, la voix rauque. À noter : the white crow = le merle blanc; to eat crow (U.S.) : avaler des couleuvres.

CUCKOO - Le coucou a la réputation de "faire cocu " les autres oiseaux, parce qu'il pond ses oeufs dans leur nid. Ce symbole est maintenant vieilli en SF et en SA (sans doute du fait qu'on ne fait plus le rapprochement des mots cocu et cuckold avec le nom de l'oiseau). En SA, le symbole de niaiserie tend aussi à disparaître, au profit du symbole actuellement très courant, celui de la folie (to go cuckoo $=$ perdre la boule). 
$D O D O$ - Le dronte, oiseau d'une espèce disparue il y a plus de deux siècles, symbolise en SA une personne peu intelligente ou amorphe, aux idées d'un autre âge : as dead as the dodo. En français, on peut rendre le mot par dinosaure. On trouve dodo dans le dialecte de Franche-Comté, dans le sens de "nigaud".

DOVE - La colombe est le symbole de la paix, en SA et en SF. Pendant la guerre du Vietnam, on a opposé les doves (pacifistes) aux hawks (partisans de la guerre). On trouve aussi as mild as a dove. Aux États-Unis, a dove-party est une réunion intime de femmes de pasteur.

$D U \boldsymbol{C K}$ - Le canard symbolise plutôt la claudication en SF, et plutôt l'aisance dans l'eau en SA. A lame duck = un raté, un canard boîteux. Duck (ou duckie) est aussi un terme affectueux : "you're a duck».

$E A G L E$ - L'aigle symbolise, en SA et en SF, la majesté et la domination, et en plus, en $\mathrm{SA}$, le regard perçant (eagle-eyed correspond à oeil de lynx).

FOWL - L'ancien nom générique de l'oiseau, qui a subi une restriction sémantique réduisant son emploi au gibier à plume, est peu usité en SA. On trouve toutefois drunk as a fowl (cf. fr. soûl comme une grive).

GANDER - Le jars, absent en SF, symbolise la sottise en SA. As stupid as a gander peut se rendre par bête comme une oie.

GOOSE - En SF et en SA, l'oie symbolise la sottise. À noter : goose-flesh = chair de poule; the goose that lays the golden eggs = la poule aux oufs d'or.

GULL - La mouette est absente en SF. Le nom anglais de cet oiseau se trouve avoir la même forme qu'un autre mot, sans rapport étymologique avec lui, et signifiant "dupe " comme nom et « duper " comme verbe (d'où gullible). La confusion n'est donc pas surprenante, mais il s'agit d'une fausse symbolique. Malgré tout, on peut rendre le nom par pigeon et le verbe par pigeonner.

$H A W K$ - Le faucon symbolise en SF et en SA la vue perçante. Par ailleurs, en SA, il symbolise la rapacité (comme vautour en français). Depuis la guerre du Vietnam, $H A W K$ symbolise aussi le caractère belliqueux (comme faucon en français).

$H E N$ - En SF, la poule symbolise la femme facile, la prostituée, mais en SA elle symbolise la femme en général (hen-party, henpecked husband). Mère-poule peut se rendre littéralement par mother-hen $(=$ motherly mum). Par contre, comme terme affectueux, ma poule ne peut pas être rendu par hen, mais par chick, chicken, duck, duckie.

$J A C K D A W$ - Le choucas, absent en SF, symbolise en SA la vanité et le faux-semblant, dans l'expression jackdaw in peacock's feathers $(=$ le geai paré des plumes du paon).

$J A Y$ - Sauf dans l'expression précédente, le geai est absent en SF. En SA, il a symbolisé la frivolité, mais, de nos jours, c'est un symbole relativement peu utilisé du bavardage (comme la pie), et aussi de la sottise naïve, de la jobardise. $A$ jaywalker est un piéton " tête en l'air " ou indiscipliné.

LARK — L'alouette symbolise, en SA plus qu'en SF, le lever du jour (rise with the lark), et en plus, en SA, la gaîté : merry (blithe, cheerful) as a lark, comme le pinson en SF. 
MAGPIE - La pie, en SF et en SA, symbolise, d'une part, le bavardage (chatter like a magpie = jaser comme une pie) et, d'autre part, le tempérament pillard : as thievish as a magpie.

NIGHTINGALE - En SF et en SA, le rossignol symbolise le don du chant (essentiellement pour la femme).

OSTRICH - En SF et en SA, l'autruche symbolise, d'une part, l'irréalisme (ostrich policy, head-in-the-sand policy) et, d'autre part, la capacité d'avaler et de digérer n'importe quoi : avoir un estomac d'autruche $=$ have the digestion of an ostrich.

$O W L$ - Peu utilisé en SF, où il symbolise quelquefois la laideur (peut-être par opposition à chouette, avec un calembour sur ce mot), le hibou, en SA, symbolise curieusement à la fois la sagesse ( $a$ wise old owl, an owlish air of wisdom) et la sottise (as stupid as an owl, to look like a stuffed owl). On trouve aussi as drunk as a (boiled) owl.

$P A R R O T$ - En SF et en SA, le perroquet symbolise le bavardage, la répétition automatique : to prate like a parrot.

PARTRIDGE - Absente en SF, la perdrix symbolise le léger embonpoint (as plump as a partridge $=$ dodu comme une caille).

$P E A C O C K$ - En SF et en SA, le paon symbolise, d'une part, la coloration excessive, et, d'autre part, la fierté, l'ostentation, la vanité (d'où peacockery = suffisance ; to peacock about = se pavaner - du latin pavo, - onis = paon).

PELICAN - Une tradition (non fondée dans les faits et plus littéraire que lexicalisée) fait du pélican, en SA et en SF, le symbole du dévouement, de l'abnégation.

PIGEON - Le pigeon symbolise couramment en SF la dupe, la victime d'une malhonnêteté, moins fréquemment en SA, où il symbolise en plus la couardise (pigeonhearted; cf. fr. capon, variante de chapon).

PLOVER - Absent en SF, le pluvier symbolise la frugalité en SA : live like a plover = vivre de l'air du temps.

POPINJAY - Ancien nom du perroquet (encore utilisé en anglais dialectal dans le sens de "pivert"), le mot est resté au sens métaphorique pour symboliser la fatuité.

QUAIL - La caille apparaît en français dans chaud comme une caille. En SA, et moins couramment en SF, il symbolise le léger embonpoint.

RAVEN - Symbolisant en SA et en SF la noirceur physique (raven locks, raven's wing), le corbeau est également, dans les deux cultures, un oiseau de mauvais augure. De plus, il symbolise couramment en SA, et moins souvent en SF, l'enrouement, la voix rauque. (Le verbe raven et l'adjectif ravenous - dont le $a$ est bref - dérivent du latin rapina et n'ont aucun rapport avec le nom de l'oiseau).

ROBIN REDBREAST - Le rouge-gorge, absent en SF, apparaît en SA, mais assez rarement, pour symboliser le caractère non méfiant.

SPARROW - En SA et en SF, le moineau symbolise l'effronterie (as cheeky as a cocksparrow) et le tempérament pillard. Le caractère peureux apparait aussi, mais surtout en SA (to startle like a frightened sparrow). À noter aussi : as shabby as a (tailless) sparrow.

$S \boldsymbol{W} A N$ - Symbole de grâce, en SF et en SA, le cygne s'oppose à l'oie dans all his geese are swans, qui n'a pas d'équivalent français, mais rappelle la fable de La Fontaine 
l'Aigle et le Hibou. Swan-song et chant du cygne sont des calques de l'allemand Schwanengesang.

THRUSH - La grive n'apparaît guère en SF que dans soûl comme une grive et à défaut de grives on mange des merles. En anglais, le mot THRUSH désigne, en fait, plusieurs oiseaux chanteurs, comme la grive commune (proprement throstle), le rossignol ou le merle. Il symbolise le chant ou le sifflement harmonieux : to whistle (ou sing) like a thrush (cf. fr. siffler comme un merle; chanter comme un rossignol).

TURKEY-COCK - Le dindon, en SF et en SA, symbolise, d'une part, la couleur rouge vif, et, d'autre part, l'insolence, l'arrogance, la vanité : to swell like a turkey-cock = gonfler le jabot. Il symbolise aussi, en SF, l'homme stupide. Dans être le dindon de la farce, il symbolise la dupe, la victime ridicule (se rend par to be made a fool of). La femelle, turkey-hen, est absente en SA, alors que la dinde symbolise la sottise féminine en SF.

TURTLE-DOVE - En SF et SA, la tourterelle symbolise l'amour juvénile et la fidélité conjugale : a couple of turtle-doves = deux tourtereaux. Dans as true as a turtle to her mate, turtle est la forme ancienne de turtle-dove : il signifie donc " tourterelle " et non pas "tortue de mer".

VULTURE - En SA et en SF, le vautour symbolise la rapacité, la voracité. À noter aussi le composé humoristique culture-vulture : "person eager to acquire culture".

\section{POISSONS, MOLLUSQUES, REPTILES, BATRACIENS, CRUSTACÉS, ANNELIDES}

$A D D E R$. - Le mot vipère correspond à deux mots anglais, $A D D E R$ et VIPER. $A D$ $D E R$ désigne la vipère commune et apparaît dans la comparaison lexicalisée as deaf as an adder, où il symbolise la surdité, mais il n'est pas utilisé métaphoriquement dans une expression du type "*she's an adder». Par contre, VIPER, qui est utilisé aussi au sens propre, peut être utilisé dans une expression du type "she's a viper", au sens métaphorique de "malignant and treacherous person"; c'est-àdire que VIPER symbolise en SA la malfaisance et la traîtrise en général, alors qu'en SF cet animal symbolise la malfaisance plus spécialement sous la forme du bavardage calomnieux.

CHAMELEON - En SA et en SF, le caméléon symbolise l'inconstance, la versatilité. $C L A M$ - La palourde est le mollusque bivalve que l'on appelle clovisse dans la région méditerranéenne, mot dérivé du provençal claus (= "qui se ferme "). Absente en $\mathrm{SA}$, la palourde symbolise en SF le caractère renfermé, le mutisme (cf. aussi US : clam up! = la ferme !).

$\boldsymbol{C R} \boldsymbol{A B}$ - Le mot anglais qui désigne le crabe a un homonyme qui désigne une pomme aigre. Il y a eu manifestement confusion, puis fusion, des deux symboliques correspondantes. En $\mathrm{SA}, C R A B$ symbolise l'aigreur de caractère : $a$ crab $=$ " $a$ sour person"; to crab = "critiquer de façon défavorable et grincheuse "; crabbed = grincheux, revêche. Comme en SF, il symbolise aussi la progression en diagonale, la marche de travers : crabwise, crabways.

CROCODILE - En SA comme en SF et ailleurs, le crocodile apparaît surtout dans le composé crocodile tears (= larmes de crocodile), symbole de l'hypocrisie. 
EEL — En SA et en SF, l'anguille symbolise le caractère insaisissable : as slippery as an eel.

FISH - En SA et en SF, le poisson symbolise le fait de bien nager : swim like a fish. Par ailleurs, en SA, il symbolise le mutisme : as dumb (ou mute) as a fish (à rendre par muet comme une carpe). Enfin, en SA, il symbolise le fait de boire beaucoup : drink like a fish = boire comme un trou. À noter aussi : a queer fish = un drôle d'oiseau; I have other fish to fry = j'ai d'autres chats à fouetter; fishy = louche (c'est-à-dire insaisissable comme un poisson).

FROG - La grenouille symbolise le contact froid en SA et, moins couramment, en SF : avoir du sang de grenouille. De plus, en SA, elle symbolise l'enrouement, la voix rauque : to have a frog in one's throat $=$ avoir un chat dans la gorge.

HERRING - En SF, le hareng symbolise la maigreur (sec comme un hareng = as thin as a rake) et la promiscuité (serrés comme des harengs = packed like sardines). En SA, HERRING a symbolisé l'épuisement, physique plutôt que moral : dead as a shotten herring (shotten $=$ "après la ponte "), mais l'expression est très vieillie. Actuellement, il symbolise surtout en SA le fait d'être salé : as salt as a herring.

LEECH - En SA et en SF, la sangsue symbolise, d'une part, la personne importune, " collante ", le " crampon ", et, d'autre part, mais plus rarement en SF, la personne insatiable ou le profiteur.

LIMPET - Absente en SF, la patelle symbolise en SA la personne "qui s'accroche", dont on ne parvient pas à se débarrasser, par exemple le fonctionnaire inamovible, ancré dans son poste. $L E E C H$ et $L I M P E T$ s'emploient souvent dans une comparaison en like, après les verbes stick et cling.

LIZARD - En SA et, plus souvent, en SF, le lézard symbolise la paresse, le "farniente ", plus spécialement au soleil. À noter : a lounge-lizard = un gigolo.

LOBSTER - Pratiquement absent en SF, le homard symbolise en SA la couleur rouge vif : as red as a (boiled) lobster = rouge comme une écrevisse.

MACKEREL - Le maquereau symbolise en SA l'aspect tacheté : mackerel sky = ciel pommelé. En français, le mot est utilisé couramment au sens de "souteneur, proxénète " (cf. aussi barbeau).

OYSTER - Assez rare en SF, l'huître y symbolise parfois la sottise (" quelle huître ! "), et, moins rarement, le bâillement (bâiller comme une huître). Ce dernier symbole apparaît aussi en SA (to gape like an oyster), où l'huître symbolise en plus le mutisme (as dumb as an oyster) et le caractère renfermé, aussi bien dans une comparaison (as close as an oyster) que dans un emploi métaphorique (" he's a regular oyster").

RATTLER - Ce mot désigne, en anglais d'Amérique, le crotale, ou serpent à sonnettes, que l'anglais britannique désigne par rattle-snake. Il symbolise le "sale type " aux Etats-Unis. Le mot side-winder désigne un petit crotale américain à progression latérale, porteur du même symbole.

ROACH - En SA et en SF, le gardon symbolise la bonne santé : as sound as a roach = frais comme un gardon. 
SARDINE - En SA et en SF, les sardines (en boîte) symbolisent, comme les harengs en $\mathrm{SA}$, la promiscuité dans un espace réduit : packed like sardines $=$ serrés comme des sardines en boîte.

SERPENT - Voir SNAKE.

SHARK - En SA et en SF, le requin symbolise la cupidité cruelle du financier retors, de l'escroc, de l'aigrefin, de l'usurier, de l'exploiteur (cf. "un requin de la finance "). Aux États-Unis, SHARK symbolise aussi l'étudiant brillant (qui accapare les meilleurs résultats) : " he's a shark at maths" = "c'est un as en maths".

SLUG - En SA et en SF, la limace symbolise la lenteur, l'indolence (d'où sluggish).

SNAIL - En SA et en SF, l'escargot symbolise la lenteur : at a snail's pace (cf. aussi l'expression humoristique allemande mit der Schneckenpost fahren).

$S N A K E$ - Le mot serpent correspond à deux mots anglais : SNAKE et SERPENT. Le second est d'un niveau de langue littéraire et désigne plutôt les gros serpents. La symbolique des deux mots se recouvre en grande partie, mais pas en totalité. Surtout sous la forme $S N A K E$, le serpent, comme en SF, symbolise le contact froid (cf. aussi $F R O G, T O A D$ ) et le mouvement sinusoïdal (to snake = serpenter et, en parlant d'un parachute, se mettre en torche). À noter aussi : to see snakes = " avoir une crise de delirium tremens". Sous la forme SNAKE ou SERPENT, cet animal, comme en SF, symbolise la traîtrise insinuante, la perfidie : snaky tongue, a snake in the grass, to cherish a serpent in one's bosom, as sharp as a serpent's tooth. Sous la forme SERPENT, cet animal symbolise, d'une part, en SA comme en SF, le diable, l'esprit du mal, et, d'autre part, surtout en SA, la sagesse et la subtilité, deux qualités qui lui sont attribuées respectivement dans St-Mathieu ("wise as serpents ", d'où serpentine wisdom) et dans la Genèse.

TOAD - En SA et en SF, le crapaud symbolise, d'une part, le contact froid, et, d'autre part, la laideur et, par suite, le dégoût qu'elle peut inspirer. D'où son emploi pour désigner une personne physiquement ou moralement repoussante : "he's a toad", "he was treated like a toad under the harrow" (= "traité plus bas que terre"); " he leads a toad-under-the-harrow existence" = "il est rejeté par tout le monde". Toad-eater (littéralement "mangeur de crapauds ") désigne un sycophante, d'où toad-eating, adj. (= servile) et toady, $\mathbf{N}$ et V (flagorneur, flagorner) : to toady to sb. $=$ lécher les bottes de qn.

TORTOISE - En SA et en SF, la tortue de terre symbolise la lenteur : at a tortoise gait $=\grave{a}$ pas de tortue, ou de sénateur. Beaucoup plus rarement, et seulement en SA, la tortue est aussi symbole de chasteté.

TROUT - En SA (et rarement en SF), la truite est, comme le gardon, symbole de bonne santé, de vivacité : as sound (ou as whole) as a trout.

TURTLE - La tortue de mer n'est pas porteuse de symbole à proprement parler, mais apparait dans l'expression to turn turtle = chavirer.

VIPER - Voir ADDER.

WORM - En SA, plus couramment qu'en SF, le ver de terre symbolise l'humilité servile et le caractère insignifiant, minable, méprisable, voire abject : " he's a worm of a man" = "c'est un minable "; " you worm !" = " misérable! ". De plus, en SA, il symbolise le souci qui ronge, le remords qui tourmente : the worm of conscience. Le verbe worm a le sens de «s'insinuer 》: « to worm oneselfinto sb's favour ». À noter également : $a$ can of worms $=$ " un vrai guêpier »; even $a$ worm will turn $=l a$ patience a des limites. 


\section{INSECTES, ARACHNIDES}

$A N T$ - En SA et en SF, la fourmi symbolise le travail zélé, incessant et minutieux (fr. un travail de fourmi). Couramment en SF, et plus rarement en SA, la fourmi symbolise aussi le caractère économe et prévoyant. Au pluriel, en SA et en SF, le mot symbolise la foule grouillante.

$B E E$ - En SF et en SA, l'abeille symbolise le travail affairé, l'activité diligente (angl. as busy as a bee). Au pluriel, ou sous la forme swarm (= essaim), le mot symbolise, en SA plus qu'en SF, le grand nombre, la foule grouillante ( $a$ swarm of children = une ribambelle d'enfants). De plus, en SA seulement, il symbolise l'idée fixe, la manie ; to have a bee in one's bonnet = avoir une marotte.

BEETLE - Ce mot est le terme générique désignant les coléoptères. L'équivalent est absent en SF. En SA, le mot symbolise la myopie (as blind as a beetle = myope comme une taupe), plus rarement la surdité (as deaf as a beetle), et enfin l'ennui (as dull as a beetle = ennuyeux comme la pluie, triste comme un jour sans pain, comme un bonnet de nuit).

$B U G$ - Ce mot, qui désigne la punaise en Grande-Bretagne, a un emploi plus large aux États-Unis où il désigne toute sorte d'insectes. On le trouve surtout dans as snug as a bug in a rug, où il symbolise le confort, la tranquillité (= approximativement : comme un coq en pâte, heureux comme un pou sur une bosse).

BUTTERFLY - En SA et en SF, le papillon (diurne) symbolise la légèreté physique, d'une part, et la vie insouciante, la frivolité, d'autre part (to butterfly = papillonner). De plus, en SA, mais moins fréquemment, il symbolise la coloration excessive.

CICADA - La cigale symbolise en SF l'insouciance, l'irréalisme, la "vie d'artiste ". Ce symbole n'est que peu utilisé en SA : l'animal lui-même est très peu connu en Grande-Bretagne, où 'il est plus ou moins confondu avec la sauterelle.

CRICKET (ou GRIG) - Pratiquement absent en SF, le grillon symbolise la gaîté un peu folle, la drôlerie : merry (ou lively) as a grig.

FLEA - Symbolisant la petitesse en SF, comme MIDGE en SA, la puce n'apparaît guère en SA que dans flea-bite, symbolisant un désagrément sans importance.

FLY - La mouche n'a pas grande valeur symbolique, ni en SA ni en SF, si ce n'est pour la faculté qu'elle a de mourir facilement et en grand nombre : to die like fies = tomber comme des mouches. Toutefois, le mot apparaît dans différentes expressions comme : a fly in the ointment = une ombre au tableau; the fly on the wheel $=$ la mouche $d u$ coche; there are no flies on him = il n'est pas né de la dernière pluie.

GADFLY - Le taon, absent en SF, symbolise en SA le caractère agaçant : a gadfly = "an irritating or harassing person".

GNAT - Le cousin, absent en SF, symbolise en SA un désagrément sans importance (= "insignificant annoyance").

GRASSHOPPER - La sauterelle a une très faible valeur symbolique. En SA, elle symbolise quelquefois la vieillesse. En SF, on désigne par l'expression "une grande sauterelle " une jeune fille dégingandée et un peu écervelée.

GRIG - Voir CRICKET. 
INSECT - En SA et en SF, le terme général d'insecte symbolise l'insignifiance ("insignificant or contemptible person or creature").

LOCUST - Le criquet, pratiquement absent en SF, symbolise en SA, au singulier ou au pluriel, le caractère dévastateur ("person of devouring or destructive propensities"). Il symbolise aussi, au pluriel, la foule grouillante.

LOUSE - En SF, et plus couramment en SA, le pou symbolise le dégoût que l'on ressent pour une personne repoussante ou méprisable, et, en SA, méchante ou mal intentionnée (un vieux pou = " un vieillard un peu repoussant "; " he's a louse » = "c'est une peau de vache ". C'est surtout sous la forme de l'adjectif qu'apparaît la valeur symbolique : lousy, comme fr. pouilleux, ou miteux, équivaut à bad et symbolise la mauvaise qualité : lousy weather = temps de chien, to feel lousy = être mal fichu.

MIDGE - Le moucheron, pratiquement absent de SF, symbolise la petitesse physique, comme la puce - ou éventuellement le moustique - en SF. D'où midget, qui désigne une personne ou une chose de très petite taille.

MOTH - Le papillon de nuit n'existe en SF que par assimilation, d'ailleurs rare, à " papillons noirs " (= " accès de mélancolie "). En SA, il symbolise le fait de tourner dangereusement autour d'un objet tentant : "person hovering around temptation » (cf. fr. "il va se brûler les ailes").

NIT - La lente, ou cuf de pou, absente en SF, symbolise la stupidité en SA : a nitwit = un débile. Nit-picking symbolise le caractère chicanier : "stop nit-picking" = " arrête de chercher des poux " (ou "de couper les cheveux en quatre").

SPIDER - Pratiquement absente en SF (sauf dans avoir une araignée au plafond), l'araignée est assez rare en $\mathrm{SA}$, où elle symbolise parfois la ruse.

TICK - Rare en SF, où elle peut symboliser le caractère "collant ", la tique symbolise en SA une personne désagréable ou méprisable. On trouve aussi en anglais d'Amérique as full as a tick = plein comme un œuf.

$\boldsymbol{W A S P}$ - N'apparaissant guère en SF que dans l'expression avoir une taille de guêpe (= to be wasp-waisted), la guêpe est surtout symbole, en SA, d'agressivité : angry as a wasp. L'adjectif waspish peut se rendre par acerbe, hargneux. À noter : un guêpier = a hornet's nest (littéralement " un nid de frelons").

Ces quelques pages n'auront sans doute pas appris grand chose aux traducteurs chevronnés : " ce n'est pas aux vieux singes qu'on apprend à faire des grimaces". Mais elles n'auront pas été inutiles, si elles ont piqué l'intérêt d'étudiants, ou de traducteurs débutants, qui, d'ailleurs, éprouveront peut-être le besoin de compléter la liste des animaux, en partant, cette fois, de la symbolique du français, d'étoffer l'analyse, de chercher à transposer les exemples non traduits, et aussi d'étendre la comparaison avec la symbolique d'autres langues que nous avons esquissée à propos des mammifères. Nous terminerons par un certain nombre d'observations que nous avons faites au cours de cette étude.

En ce qui concerne la forme des comparaisons lexicalisées, on remarque le goût de la langue anglaise pour l'allitération, qui parfois semble « forcer " un peu la symbolique : have bats in the belfry, blind as a bat, blind as a beetle, blind as a buzzard (la buse est-elle 
myope à ce point ?), dead as the dodo, act the giddy goat, mute as a mouse, as snug as a bug in a rug, etc. Le français est beaucoup moins enclin à ce jeu phonétique : si l'on traite de " moule " quelqu'un de " mou ", il s'agit sans doute davantage de l'influence de la paronymie (comme pour police/poulet) que du goût pour l'allitération. L'homonymie - on l'a vu avec crab et gull - joue parfois un rôle, elle aussi.

On aura pu noter, par ailleurs, la présence sous-jacente de l'arrière-plan culturel. Les traditions religieuses et littéraires, sans être nécessairement à l'origine d'un symbole, peuvent s'en nourrir et le renforcer en échange. L'association du serpent avec les forces du mal est une tradition commune à tous les pays de culture judéo-chrétienne. Dans le Roman de Renart, le lièvre s'appelle Couart, le lion Noble, et Renart est le personnage rusé. La cigale et la fourmi doivent certainement la pérennité de leur symbolique à la fable de La Fontaine, comme les moutons (de Panurge) à l'œuvre de Rabelais. Le cinéma peut, d'ailleurs, prendre le relais de la littérature : le mythe du Grand méchant loup doit davantage sa popularité à Walt Disney qu'à Simon Goodenough. Quant à l'inclusion du dalmatien dans la symbolique anglaise, il est vraisemblable sinon qu'elle soit due directement au film de Walt Disney, du moins qu'elle ait été grandement facilitée par le succès de ce dessin animé.

L'histoire sociale - ou l'histoire tout court - a également une influence sur la symbolique : si la cruauté du loup est restée, pour les Français et les continentaux en général, une effrayante réalité jusqu'au début du $\mathrm{XX}^{\mathrm{e}}$ siècle, les Britanniques y sont évidemment moins sensibles, puisque le loup a disparu d'Angleterre avant la fin du XVIe siècle, d'Écosse avant la fin du XVII et d'Irlande avant la fin du XVIII'. Quant au dronte, ou dodo, sa symbolique est due directement à sa disparition. Des faits sociologiques même récents peuvent avoir une influence non négligeable : par exemple, l'utilisation, en France, de l'écureuil comme image publicitaire de la Caisse d'épargne a manifestement renforcé la valeur symbolique de cet animal.

Il arrive aussi que la symbolique se limite à une micro-communauté. C'est ainsi que les habitués de la bourse utilisent des métaphores très spécialisées : bear (spéculateur à la baisse), bull (spéculateur à la hausse), stag ("person who applies for shares of new issue with a view to selling at once at a profit "). L'humour n'est sans doute pas absent de ces emplois pittoresques.

On observe, il est vrai, que la symbolique animale semble souvent participer de l'activité ludique. On trouve même, à la frontière du discours et de la langue, un certain nombre d'expressions où l'humour (ou l'ironie) se fonde sur l'antiphrase : le jeu consiste à attribuer à un animal la caractéristique inverse de celle qu'on lui connaît. D'où des expressions non lexicalisées du type "as comely (ou as nimble) as a cow in a cage " (où l'allitération est également sensible), "as slender in the middle as a cow in the waist " (= " mince comme une baleine "), " to dance like an elephant" (= "danser comme une lessiveuse "), "as bold as an Essex lion" (où Essex lion désigne un veau), "as like as an apple to an oyster", etc. Un jeu de mots peut d'ailleurs ajouter au comique : dans « to swim like a tailor's goose », goose désigne non pas une oie, mais un fer à repasser dont le manche a la forme d'un col de cygne; on peut rendre l'expression par "nager comme un chien de fusil ».

Enfin, les lectrices n'auront pas manqué de remarquer le caractère quelque peu misogyne de la symbolique animale, indépendamment de la langue. Les rubrique BITCH, CAT, COW, GOAT, HORSE, SHREW, SOW, WHALE, GOOSE, TURKEYCOCK, GRASSHOPPER donnent des illustrations de cette tendance. L'auteur n'y est évidemment pour rien. Le M.L.F. ne pourra guère s'en prendre qu'à ce qu'il est convenu d'appeler " la sagesse populaire ", dont on sait déjà que la vision du monde qu'elle implique n'est pas exempte de machisme. 
Il reste à se demander si la symbolique animale constitue un élément figé du lexique, puisqu'on ne crée pas de nouvelles espèces et que les espèces existantes ont des caractéristiques connues et non susceptibles d'évolution. À étudier les choses de près, on remarque que des exemples relativement récents, datant des années 1960 et déjà lexicalisés, apportent la preuve qu'un certain développement est possible. Ainsi, mule est apparu comme symbolisant la contrebande (a mule $=$ "an individual who smuggles or delivers illicit drugs"). De même, albatross (par allusion au poème de Coleridge) est apparu comme symbolisant le fardeau que fait peser sur la vie ou la conscience une dette, une responsabilité, une faute commise, notamment dans l'expression an albatross around one's neck. Affirmer que la symbolique animale est un "ensemble fini " serait compter sans l'évolution de la société elle-même et, surtout, sans la richesse de l'imagination humaine.

* Cet article reprend une communication faite au XXVe Congrès de la Société des anglicistes de l'enseignement supérieur, Caen, 10-12 mai 1985. 